Published in final edited form as:

Leuk Lymphoma. 2019 April ; 60(4): 1023-1029. doi:10.1080/10428194.2018.1516036.

\title{
A Comparison of Patients with Acute Myeloid Leukemia and High-Risk Myelodysplastic Syndrome Treated On versus Off Study
}

\author{
SA Buckley ${ }^{\# 1}$, ME Percival ${ }^{\# 2,3}$, M Othus $^{4}$, AB Halpern ${ }^{2,3}$, EM Huebner $^{3}$, PS Becker $^{2,3}$, C \\ Shaw $^{3}$, M Shadman ${ }^{2,3}$, RB Walter ${ }^{2,3,5}$, and EH Estey ${ }^{2,3}$ \\ ${ }^{1}$ Hematology/Oncology Fellowship Program, University of Washington, Seattle, WA \\ ${ }^{2}$ Department of Medicine, University of Washington, Seattle, WA, USA \\ ${ }^{3}$ Clinical Research Division, Fred Hutchinson Cancer Research Center, Seattle, WA, USA \\ ${ }^{4}$ Public Health Sciences Division, Fred Hutchinson Cancer Research Center, Seattle, WA, USA \\ ${ }^{5}$ Department of Epidemiology, University of Washington, Seattle, WA, USA \\ \# These authors contributed equally to this work.
}

\begin{abstract}
Patients with newly diagnosed (ND) and relapsed/refractory (RR) acute myeloid leukemia (AML) and high-risk myelodysplastic syndrome (MDS, $\geq 10 \%$ blasts) often receive intensive chemotherapy at diagnosis and relapse. We retrospectively identified 365 patients and categorized the reasons for receiving treatment off study (medical, logistical, or unclear). The pre-treatment characteristics of the on and off study groups were similar. Rates of complete remission (CR) without measurable residual disease were significantly higher for ND patients treated on vs. off study (61\% vs. $35 \%$ ), but CR rates and survival were low for all RR patients regardless of study assignment. The subset of ND patients treated off study for medical reasons had significantly decreased overall survival and relapse-free survival. Standard, stringent study eligibility criteria may delineate a population of ND, but not RR, patients with improved outcomes with intensive induction chemotherapy.
\end{abstract}

\section{Keywords}

AML; MDS; clinical trials; induction chemotherapy; on study

Corresponding Author: Mary-Elizabeth Percival, MD, MS. University of Washington, 825 Eastlake Ave E, Box 358081 / MS G3200, Seattle, WA 98109. Phone: 206.606.1320. Fax: 206.606.1130. mperciva@uw.edu.

Authorship Contributions All authors collected, analyzed, and interpreted data, and provided critical feedback on the manuscript. S.A.B. and M.E.P. drafted and edited the manuscript, and, along with E.H.E., conceptualized the study. M.O. performed statistical analysis. The manuscript was reviewed and approved by all authors.

Disclosure of Interest: The authors report no conflicts of interest. 


\section{Introduction}

Fit, younger individuals with acute myeloid leukemia (AML) typically undergo intensive induction chemotherapy at diagnosis and relapse.[1] Despite National Comprehensive Cancer Network recommendations,[2] only 5 to $10 \%$ of adults with AML in the United States are treated on a clinical trial.[3] There are many reasons for low enrollment, including a decentralized healthcare system leading to travel burdens,[4] financial concerns,[5] stringent inclusion/exclusion criteria, logistical problems, and physician and patient preferences. These factors lead to selection bias, limiting broad applicability of conclusions from clinical trials. In one analysis, patients excluded from participation in phase 3 trials but treated in a similar fashion had similar survival.[6] Other studies, however, have shown that patients not enrolled on trials were less fit and had worse outcomes following similar treatment. $[7,8]$ These studies are from European centers with higher trial enrollment (close to $50 \%$ in all three studies) than is common in the U.S.

Drugs not routinely employed in AML, although approved by the Food and Drug Administration (FDA), are sometimes administered via investigator-initiated studies independent of pharmaceutical company participation. These trials can test novel combinations of approved drugs. They generally provide more latitude for treating patients "off study" and formed the basis for the following effort to compare characteristics, response, and survival in patients with AML treated on study vs. off study.

\section{Methods}

Patients treated for AML or high-risk MDS ( $>10 \%$ blasts) at the University of Washington/ Fred Hutchinson Cancer Research Center between January 1, 2008 and November 15, 2015 were identified through our institutional database. This retrospective analysis was approved by the Fred Hutchinson/Cancer Consortium Institutional Review Board and conducted in accordance with the principles of the Declaration of Helsinki. Protocols for newly diagnosed (ND) and relapsed/refractory (RR) patients were included if $>15$ patients were each treated on and off study. The ND protocols were: (1) G-CSF, cladribine, cytarabine, and doseescalated mitoxantrone (ND G-CLAM),[9] and (2) idarubicin, cytarabine, and pravastatin (ND IAP).[10] The RR protocols were: (1) RR G-CLAM, (2) decitabine followed by mitoxantrone, etoposide, cytarabine (RR D-MEC),[11] and (3) G-CSF, clofarabine, cytarabine (RR G-CLAC).[12] Responses were evaluated based on European LeukemiaNet guidelines[13] and included (1) the more stringent complete remission (CR) without measurable residual disease (MRD) as $<5 \%$ morphologic blasts with peripheral blood neutrophils $\geq 1000 / \mu \mathrm{L}$, platelets $\geq 100,000 / \mu \mathrm{L}$, and no MRD by flow cytometry, conventional metaphase cytogenetics, or fluorescence in situ hybridization, and (2) the broader CR/CRi, which included CR without MRD, CR with MRD, and CRi (neutropenia and/or thrombocytopenia). We identified 401 induction attempts. Four patients treated off-protocol did not have a marrow performed to assess response, and were excluded from further analysis, leaving a total of 397 induction attempts in a total of 365 patients. 208 of these attempts (52\%) were given on study with the remaining $48 \%$ given off study for medical, logistical, or unclear reasons, as detailed below. 
Because it was at times difficult to retrospectively identify the reason for off study treatment our primary comparison was between attempts given on vs. off study. We also analyzed outcomes [response, relapse-free survival (RFS) and survival] in those treated off study. We classified the reasons patients received off study treatment as medical, logistical, or unclear in our retrospective analysis. Medical reasons leading to off study treatment includied high Treatment-Related Mortality (TRM) score[14], treatment urgency (i.e. hyperleukocytosis or leukostasis), low ejection fraction, abnormal laboratory values, and concurrent life-limiting diagnosis; logistical reasons for off study treatment included patient or physician preference, protocol not open, financial constraints, blast count too low.. Relapse was defined as morphologic increase in blasts to $25 \%$. TRM score approximates the probability of death of ND patients within 28 days of beginning intensive induction treatment. A "high" score generally corresponded to a $>10-15 \%$ probability of TRM and is similarly applicable to people with ND or relapsed/refractory AML.

Logistic regression was used to evaluate the association between baseline factors and best response after up to 2 induction cycles while the Cox model did the same for overall survival (OS) and relapse-free survival (RFS). For ND patients, covariates evaluated were age (as a continuous variable), cytogenetics (adverse vs. not using Medical Research Council criteria), [15] de novo vs. secondary disease, regimen, number of cycles to best response, and TRM score. For RR patients, additional covariates included prior allogeneic HCT, duration of first

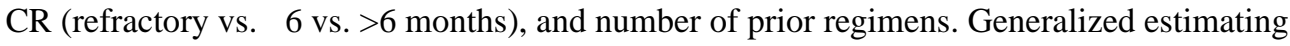
equation (GEE) methods with unstructured correlation structures were used to account for patients $(n=32)$ with data on more than one trial as needed. All authors had access to the primary data. Statistical analysis was performed by M.O.

\section{Results}

Analyses included 397 induction attempts (in 365 separate patients), including 160 for ND and 237 for RR AML with median follow-up for censored patients of 16 months (range 384) (Table 1). No significant differences in baseline characteristics were noted between patients treated on vs. off study, except for a higher TRM score in patients treated off study (ND 2.9 vs. 4.3, p=0.006; RR: 3.0 vs. 5.2, p<0.001. Compared to patients treated on study, the subset treated off study for medical reasons had higher TRM scores (ND: 2.9 vs. 4.7, $\mathrm{p}=0.006$; RR: 3.0 vs. $13.0, \mathrm{p}<0.001)$. Patients treated off study for logistical reasons were similar to those treated on study in all measured respects except that they were more likely to have undergone HCT if they were RR ( $\mathrm{p}=0.002)$. Reasons for treatment off study are summarized in Table 2.

Among ND patients, remission rates were significantly higher for patients treated on vs. off study [CR without MRD: $61 \%$ (51/83) vs. $35 \%$ (26/74), p=0.001, CR/CRi: 86\% (72/83) vs. $64 \%(48 / 75), \mathrm{p}=0.002)]$, and were particularly low for patients treated off study for medical reasons [CR without MRD: 30\% (8/28), CR/CRi 61\% (17/28)]. Among the other covariates examined only adverse cytogenetics was associated with lower rates of CR without MRD and of CR/CRi while secondary AML was similarly associated with lower CR/CRi rates (Table 3). However after accounting for these variables, odds of CR without MRD and $\mathrm{CR} / \mathrm{CRi}$ were approximately 4 -fold and 2.5 -fold higher respectively if treatment was given 
on study vs. off study for medical reasons (Table 3). In contrast, remission rates among RR patients were not significantly higher for those treated on vs. off study (CR without MRD: $26 \%$ vs. $22 \%, \mathrm{p}=0.41$; CR/CRi: $50 \%$ vs. $44 \%, \mathrm{p}=0.42$ ). Although remission rates for patients treated off study for medical reasons were particularly low (CR without MRD: 11\%; CR/ CRi: 39\%), multivariable analysis did not find this variable affected remission rates (odds ratio 0.63 for $\mathrm{CR}$ w/o MRD, 1.41 for $\mathrm{CR} / \mathrm{CRi}$, p-values $0.59,0.63$ ) certainly at least to the same extent as was observed in ND patients (Table 3).

Time-to-event outcomes among ND patients treated on vs. off study indicated survival and RFS were similar comparing on study vs off study for any reason patients (median OS: 22 vs. 21 months, $\mathrm{p}=0.17$ and RFS: 19 vs. 16 months, $\mathrm{p}=0.32$ ). However, those treated off study for medical reasons had significantly poorer OS (median 8 months) and RFS (median 7 months) (Figure 1 and Supplemental Figure). Survival outcomes for those treated off study for logistical reasons as well were most similar to those treated on study (Figure 1). Multivariable analysis confirmed these results: ND patients treated on vs. off study had similar OS and RFS, though those treated off study for medical reasons had approximately 2 to 3-fold higher risk of death (OS) or RFS than patients treated on study (Table 4). On and off study RR patients had similar OS (median 8 vs. 7 months, $\mathrm{p}=0.46$ ) and RFS (median 12 vs. 10 months, $p=0.79$ ), and those treated off study for medical reasons fared slightly worse though not significantly so (Figure 1), including after multivariable adjustment (Table 4).

We examined whether other effects could account for the findings we observed. In case patients who were treated off study only because the protocol was not available had better outcomes, we performed another multivariable analysis of the data excluding these 59 patients, and results remained unchanged and non-significant (data not shown). We also examined rates of transplant in patients on and off study; a cause-specific Cox regression model accounting for censoring and competing risks demonstrated no significant differences, with a HR for ND patients of 0.99 (95\% CI 0.56-1.74; $\mathrm{p}=0.96)$ and a HR for $\mathrm{R} / \mathrm{R}$ patients of 0.72 (95\% CI $0.42-1.25, \mathrm{p}=0.25$ ).

We wondered if the poorer survival outcomes in off study ND patients could be explained by treatment-related mortality or by higher rates of relapse. Four-week TRM rates for patients with ND AML were $4 \%$ for on study patients and $12 \%$ for off study patients $(\mathrm{p}=0.07)$; broken down by reason off study, the rates were $11 \%$ for medical reasons, $14 \%$ for logistical reasons, and $0 \%$ for unclear reasons. At 8 weeks, the corresponding TRM rates were $6 \%$ for on study and $13 \%$ for off study ( $\mathrm{p}=0.17$ ); broken down by reason off study, the rates were $11 \%$ for medical reasons, $17 \%$ for off for logistical reasons, and $0 \%$ for off for unclear reasons. Analysis of the competing risks of relapse and TRM without relapse showed that in neither ND nor RR patients were these risks affected by treatment on vs off study, or by treatment on study vs off study for medical reasons. We also fit multivariable logistic regression models in the subset of patients alive at day 28. In all the models we fit, the CR rate was significantly higher among patients who received therapy on study. In models that controlled for cytogenetic risk, secondary AML, and TRM score, the odds ratio (OR) for morphologic $\mathrm{CR}$ was $2.92(\mathrm{p}=0.026)$ and the $\mathrm{OR}$ for $\mathrm{CR}$ without MRD was $2.48(\mathrm{p}=0.021)$. The poorer survival outcomes in off study ND patients were not clearly explained by 
treatment-related mortality or rates of relapse, and the CR rate remained higher in on study patients when limited to patients who survived to day 28 .

\section{Discussion}

Our data suggest little difference in survival between on and off study patients given intensive induction therapies. However, the off study group is heterogeneous, and perhaps a more interesting comparison is with the subset of patients treated off study for medical reasons. Our study is limited because reasons for off study treatment were assessed retrospectively and were susceptible to misclassification.

Nonetheless, our data suggest that ND patients excluded for medical ineligibility fare particularly poorly. While this finding may seem intuitive it remained true even after accounting for potential differences in pre-treatment medical characteristics between these patients and those treated on study. This finding may simply reflect failure to account for other quantifiable medical characteristics. Many co-morbidity indices exist, but no one methodology has been adopted widely for optimal risk stratification, particularly for older patients; $[16,17,18]$ our study did not capture many of the factors captured in these indices, and one or more of those factors may be predictive of outcome. Additionally, the overall survival diverges early between the off-study medical group and others (Figure 1A), suggesting that the poor outcomes in this subset may reflect higher early treatment-related mortality that could not be fully demonstrated in our small sample size. However, our finding that medically ineligible patients fare poorly may also reflect the ability of treating physicians to subjectively, but accurately, assess which ND patients will do particularly poorly and therefore exclude them from studies, or to subconsciously be more attentive to patients treated on study. The absence of differences between medically excluded and other RR patients may reflect the poor outcomes for all patients with RR AML.

Our results suggest that current study eligibility criteria help delineate an ND, but not demonstrably an RR population, with better treatment responses. The desirability of maintaining these criteria is less clear and assumes that patients who are currently medically ineligible and thus excluded from many clinical trials would do worse if treated on these putatively promising studies than if given alternative (or no) treatment. Perhaps allowance could be made for inclusion of such patients when evaluating new treatments. Indeed, the recently published 2017 European LeukemiaNet AML guidelines encourage movement away from arbitrary age and organ function eligibility cut-offs when deciding whether older patients should receive intensive induction therapy.[13] Additionally, the American Society of Clinical Oncology now recommends liberalizing enrollment criteria for patients in oncology clinical trials, because of high rates of organ dysfunction, comorbidity, or prior malignancy in otherwise eligible patients with solid tumors.[19, 20] The generalizability of results is also limited if obtained in highly select clinical trial cohort. We suggest that RR studies should include more liberal eligibility criteria, especially since a recent brief report indicated that the response rate for phase 1 oncology studies may be higher than previously suggested.[21] 
Determining the proper balance of treatment intensity for medically less fit patients has been challenging at our center. We performed a trial with reduced doses of CPX-351 for patients with a high TRM score ( $\geq 13.1$, which corresponds roughly to a $13 \%$ chance of dying in the first 28 days after induction), which showed minimal efficacy without adequate improvement in toxicity.[22] An ongoing study at our center randomizes patients with a similarly high TRM score to full-dose G-CLAM or reduced-dose G-CLAM (NCT03012672). Additionally, a recent provocative study from MD Anderson Cancer Center specifically enrolled patients who did not meet eligibility criteria for other trials, demonstrating that treatment of such "ineligible" patients was feasible.[23] Only prospective studies with less rigid eligibility criteria can assess whether patients with co-morbidities currently precluding enrollment in most clinical trials would benefit from enrollment on such trials relative to the often unsatisfactory alternatives.

\section{Supplementary Material}

Refer to Web version on PubMed Central for supplementary material.

\section{Acknowledgments}

Funding Details

The conduct of one of the clinical trials described here was partially funded by Protocol Specific Research Support from the Fred Hutchinson/University of Washington Cancer Consortium Cancer Center Support Grant of the National Institutes of Health (P30-CA015704). S.A.B. is supported by a fellowship training grant from the National Heart, Lung, and Blood Institute/National Institutes of Health (NHLBI/NIH: T32-H007093).

\section{References}

1. Estey E. Acute myeloid leukemia: 2016 Update on risk-stratification and management. American journal of hematology. 2016 8;91(8):824-46. doi: 10.1002/ajh.24439. PubMed PMID: 27417880; eng. [PubMed: 27417880]

2. O'Donnell MR, Abboud CN, Altman J, et al. NCCN Clinical Practice Guidelines Acute myeloid leukemia. J Natl Compr Canc Netw. 2012 8;10(8):984-1021. PubMed PMID: 22878824. [PubMed: 22878824]

3. Rowe JM. Closer to the truth in AML. Blood. 20094 30;113(18):4129-30. doi: 10.1182/ blood-2008-12-192856. PubMed PMID: 19406994. [PubMed: 19406994]

4. Onega T, Duell EJ, Shi X, et al. Geographic access to cancer care in the U.S. Cancer. 20082 15;112(4):909-18. doi: 10.1002/cncr.23229. PubMed PMID: 18189295. [PubMed: 18189295]

5. Wong YN, Schluchter MD, Albrecht TL, et al. Financial Concerns About Participation in Clinical Trials Among Patients With Cancer. J Clin Oncol. 20162 10;34(5):479-87. doi: 10.1200/JCO. 2015.63.2463. PubMed PMID: 26700120; PubMed Central PMCID: PMCPMC4872013. [PubMed: 26700120]

6. Mengis C, Aebi S, Tobler A, et al. Assessment of differences in patient populations selected for excluded from participation in clinical phase III acute myelogenous leukemia trials. J Clin Oncol. 2003 11 1;21(21):3933-9. doi: 10.1200/JCO.2003.03.186. PubMed PMID: 14581417. [PubMed: 14581417]

7. Ostgard LS, Norgaard M, Sengelov H, et al. Improved outcome in acute myeloid leukemia patients enrolled in clinical trials: A national population-based cohort study of Danish intensive chemotherapy patients. Oncotarget. 201610 6. doi: 10.18632/oncotarget.12495. PubMed PMID: 27732947.

8. Stevens JM, Macdougall F, Jenner M, et al. Patterns of recruitment into acute myeloid leukaemia (AML) 15 and outcome for young patients with AML at a single referral centre. Br J Haematol. 
2009 4;145(1):40-4. doi: 10.1111/j.1365-2141.2008.07561.x. PubMed PMID: 19210510. [PubMed: 19210510]

9. Halpern AB, Othus M, Huebner EM, et al. A Phase 1/2 Study of G-CSF, Cladribine, Cytarabine, and Dose-Escalated Mitoxantrone (G-CLAM) in Adults with Newly Diagnosed Acute Myeloid Leukemia (AML) or High-Risk Myelodysplastic Syndrome (MDS). Blood. 2016:1068.

10. Shadman M, Mawad R, Dean C, et al. Idarubicin, cytarabine, and pravastatin as induction therapy for untreated acute myeloid leukemia and high-risk myelodysplastic syndrome. Am J Hematol. 2015 6;90(6):483-6. doi: 10.1002/ajh.23981. PubMed PMID: 25689471; PubMed Central PMCID: PMCPMC4803479. [PubMed: 25689471]

11. Halpern AB, Othus M, Huebner EM, et al. Mitoxantrone, etoposide, and cytarabine (MEC) following epigenetic priming with decitabine in adults with relapsed/refractory acute myeloid leukemia or other high-grade myeloid neoplasms: a phase 1/2 study. Leukemia. 2017:in press.

12. Becker PS, Kantarjian HM, Appelbaum FR, et al. Clofarabine with high dose cytarabine and granulocyte colony-stimulating factor (G-CSF) priming for relapsed and refractory acute myeloid leukaemia. Br J Haematol. 2011 10;155(2):182-9. doi: 10.1111/j.1365-2141.2011.08831.x. PubMed PMID: 21848522; PubMed Central PMCID: PMCPMC4834701. [PubMed: 21848522]

13. Dohner H, Estey E, Grimwade D, et al. Diagnosis and management of AML in adults: 2017 ELN recommendations from an international expert panel. Blood. 2017 26;129(4):424-447. doi: 10.1182/blood-2016-08-733196. PubMed PMID: 27895058; eng. [PubMed: 27895058]

14. Walter RB, Othus M, Borthakur G, et al. Prediction of early death after induction therapy for newly diagnosed acute myeloid leukemia with pretreatment risk scores: a novel paradigm for treatment assignment. J Clin Oncol. 201111 20;29(33):4417-23. doi: 10.1200/JCO.2011.35.7525. PubMed PMID: 21969499; PubMed Central PMCID: PMCPMC3221524. [PubMed: 21969499]

15. Grimwade D, Hills RK, Moorman AV, et al. Refinement of cytogenetic classification in acute myeloid leukemia: determination of prognostic significance of rare recurring chromosomal abnormalities among 5876 younger adult patients treated in the United Kingdom Medical Research Council trials. Blood. 20107 22;116(3):354-65. doi: 10.1182/blood-2009-11-254441. PubMed PMID: 20385793. [PubMed: 20385793]

16. Wass M, Hitz F, Schaffrath J, et al. Value of Different Comorbidity Indices for Predicting Outcome in Patients with Acute Myeloid Leukemia. PLoS One. 2016;11(10):e0164587. doi: 10.1371/ journal.pone.0164587. PubMed PMID: 27732646; PubMed Central PMCID: PMCPMC5061362. [PubMed: 27732646]

17. Sorror ML, Storer BE, Fathi AT, et al. Development and Validation of a Novel Acute Myeloid Leukemia-Composite Model to Estimate Risks of Mortality. JAMA Oncol. 2017 12 1;3(12):16751682. doi: 10.1001/jamaoncol.2017.2714. PubMed PMID: 28880971; PubMed Central PMCID: PMCPMC5824273. [PubMed: 28880971]

18. Klepin HD, Geiger AM, Tooze JA, et al. Geriatric assessment predicts survival for older adults receiving induction chemotherapy for acute myelogenous leukemia. Blood. 20135 23;121(21): 4287-94. doi: 10.1182/blood-2012-12-471680. PubMed PMID: 23550038; PubMed Central PMCID: PMCPMC3663423. [PubMed: 23550038]

19. Lichtman SM, Harvey RD, Damiette Smit MA, et al. Modernizing Clinical Trial Eligibility Criteria: Recommendations of the American Society of Clinical Oncology-Friends of Cancer Research Organ Dysfunction, Prior or Concurrent Malignancy, and Comorbidities Working Group. J Clin Oncol. 201711 20;35(33):3753-3759. doi: 10.1200/JCO.2017.74.4102. PubMed PMID: 28968172. [PubMed: 28968172]

20. Kim ES, Bruinooge SS, Roberts S, et al. Broadening Eligibility Criteria to Make Clinical Trials More Representative: American Society of Clinical Oncology and Friends of Cancer Research Joint Research Statement. J Clin Oncol. 201711 20;35(33):3737-3744. doi: 10.1200/JCO. 2017.73.7916. PubMed PMID: 28968170; PubMed Central PMCID: PMCPMC5692724. [PubMed: 28968170]

21. Chakiba C, Grellety T, Bellera C, et al. Encouraging Trends in Modern Phase 1 Oncology Trials. N Engl J Med. 20186 7;378(23):2242-2243. doi: 10.1056/NEJMc1803837. PubMed PMID: 29874526. [PubMed: 29874526]

22. Walter RB, Othus M, Orlowski KF, et al. Unsatisfactory efficacy in randomized study of reduceddose CPX-351 for medically less fit adults with newly diagnosed acute myeloid leukemia or other 
high-grade myeloid neoplasm. Haematologica. 2018 3;103(3):e106-e109. doi: 10.3324/haematol. 2017.182642. PubMed PMID: 29242304. [PubMed: 29242304]

23. Montalban-Bravo G, Huang X, Jabbour E, et al. A clinical trial for patients with acute myeloid leukemia or myelodysplastic syndromes not eligible for standard clinical trials. Leukemia. 2017 2;31(2):318-324. doi: 10.1038/leu.2016.303. PubMed PMID: 27795561. [PubMed: 27795561] 
A

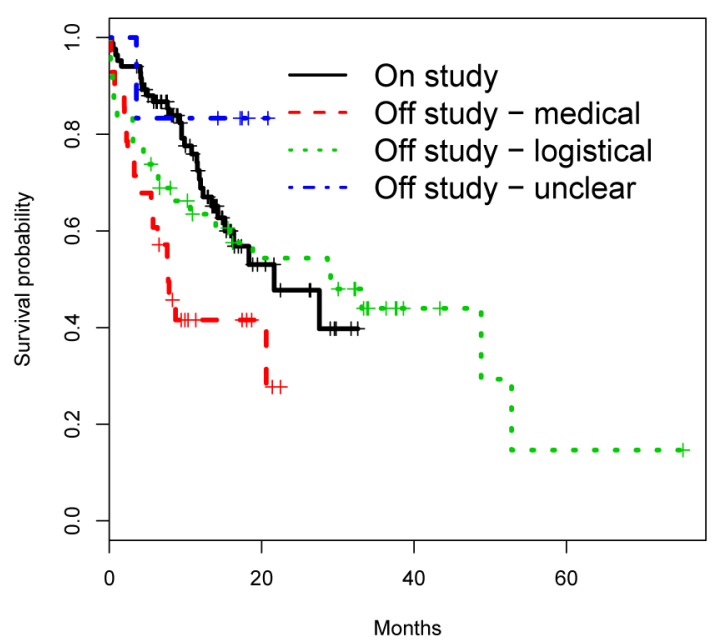

C Overall Survival - Relapsed/Refractory

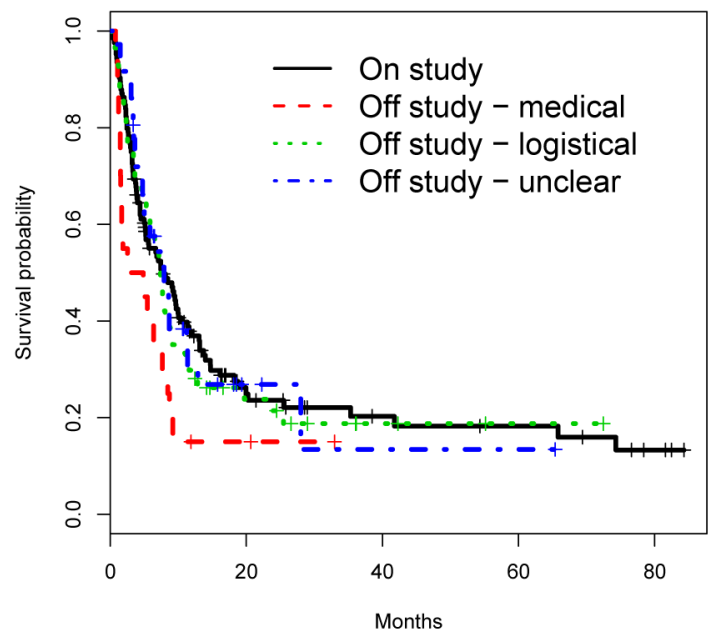

B

Relapse-free Survival - Newly Diagnosed

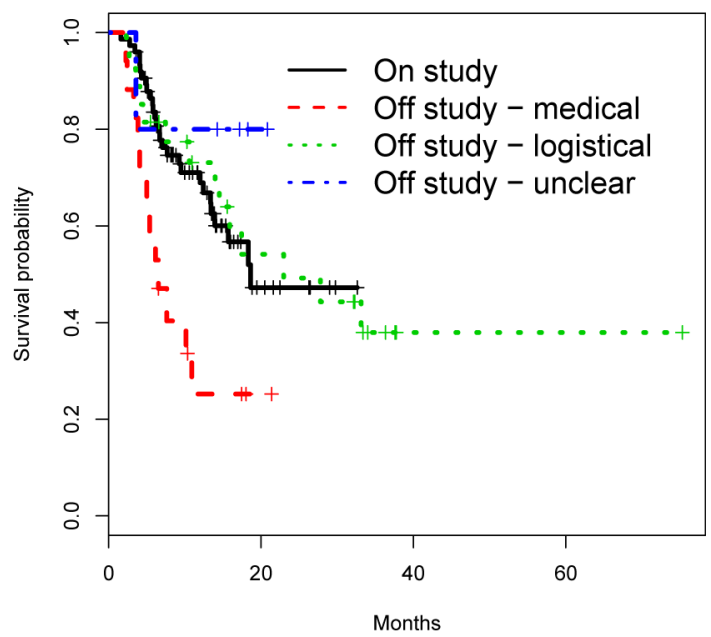

D Relapse-free Survival - Relapsed/Refractory

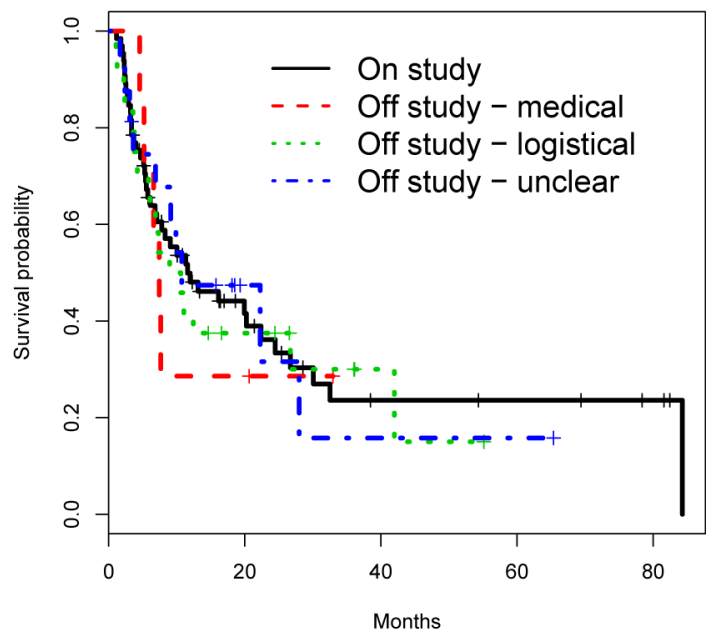

Figure 1.

Survival curves for patients treated on and off study among ND patients (a, b) and RR patients (c, d). 


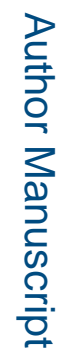

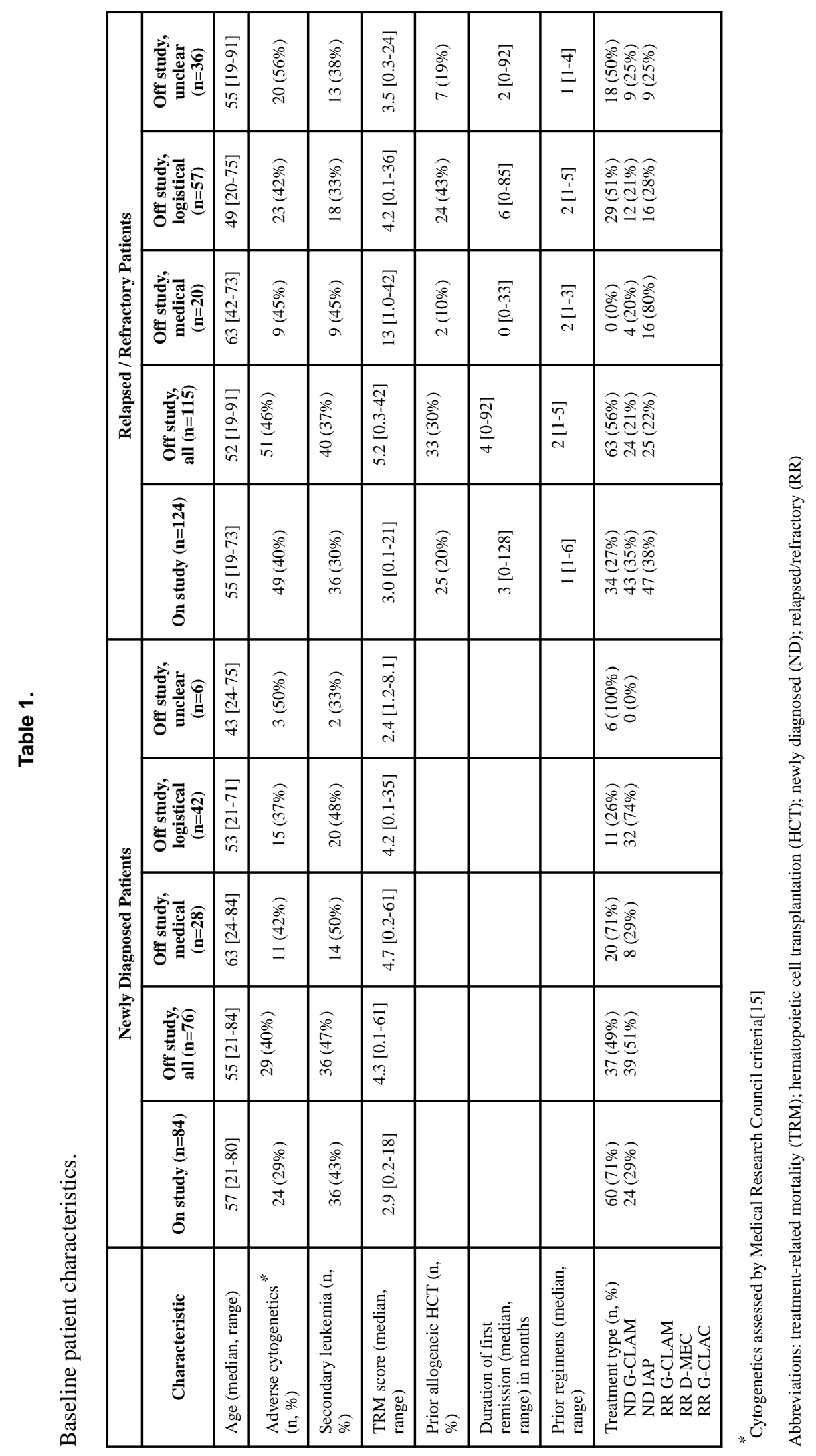

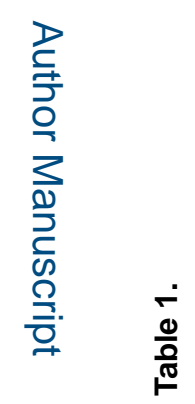

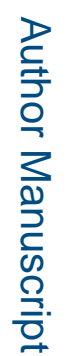

Leuk Lymphoma. Author manuscript; available in PMC 2020 April 01. 
Table 2.

Breakdown of reasons for off study treatment for newly diagnosed and relapsed / refractory patients. Percentages do not sum to $100 \%$ due to rounding.

\begin{tabular}{|lll|}
\hline \multicolumn{1}{|c}{ Reason Off Study } & $\begin{array}{c}\text { Newly diagnosed } \\
(\mathbf{n = 7 6 )}\end{array}$ & $\begin{array}{c}\text { Relapsed / } \\
\text { Refractory } \\
(\mathbf{n = 1 1 3})\end{array}$ \\
\hline Medical reason & $\mathbf{2 8 ( 3 6 \% )}$ & $\mathbf{2 0}(\mathbf{1 8 \%})$ \\
\hline High TRM score & $9(12 \%)$ & $15(13 \%)$ \\
\hline Abnormal labs or cardiac function & $8(11 \%)$ & $5(4 \%)$ \\
\hline Urgency to starting treatment & $11(14 \%)$ & $0(0 \%)$ \\
\hline Logistical reason & $\mathbf{4 2 ( 5 5 \% )}$ & $\mathbf{5 7}(\mathbf{5 0 \%})$ \\
\hline Patient or physician preference & $6(8 \%)$ & $24(24)$ \\
\hline Protocol not open & $31(41 \%)$ & $28(25 \%)$ \\
\hline Financial constraints & $5(7 \%)$ & $2(2 \%)$ \\
\hline Blast count too low & $0(0 \%)$ & $3(3 \%)$ \\
\hline Unclear reason & $\mathbf{6 ( 8 \% )}$ & $\mathbf{3 6}(\mathbf{3 2 \%})$ \\
\hline
\end{tabular}




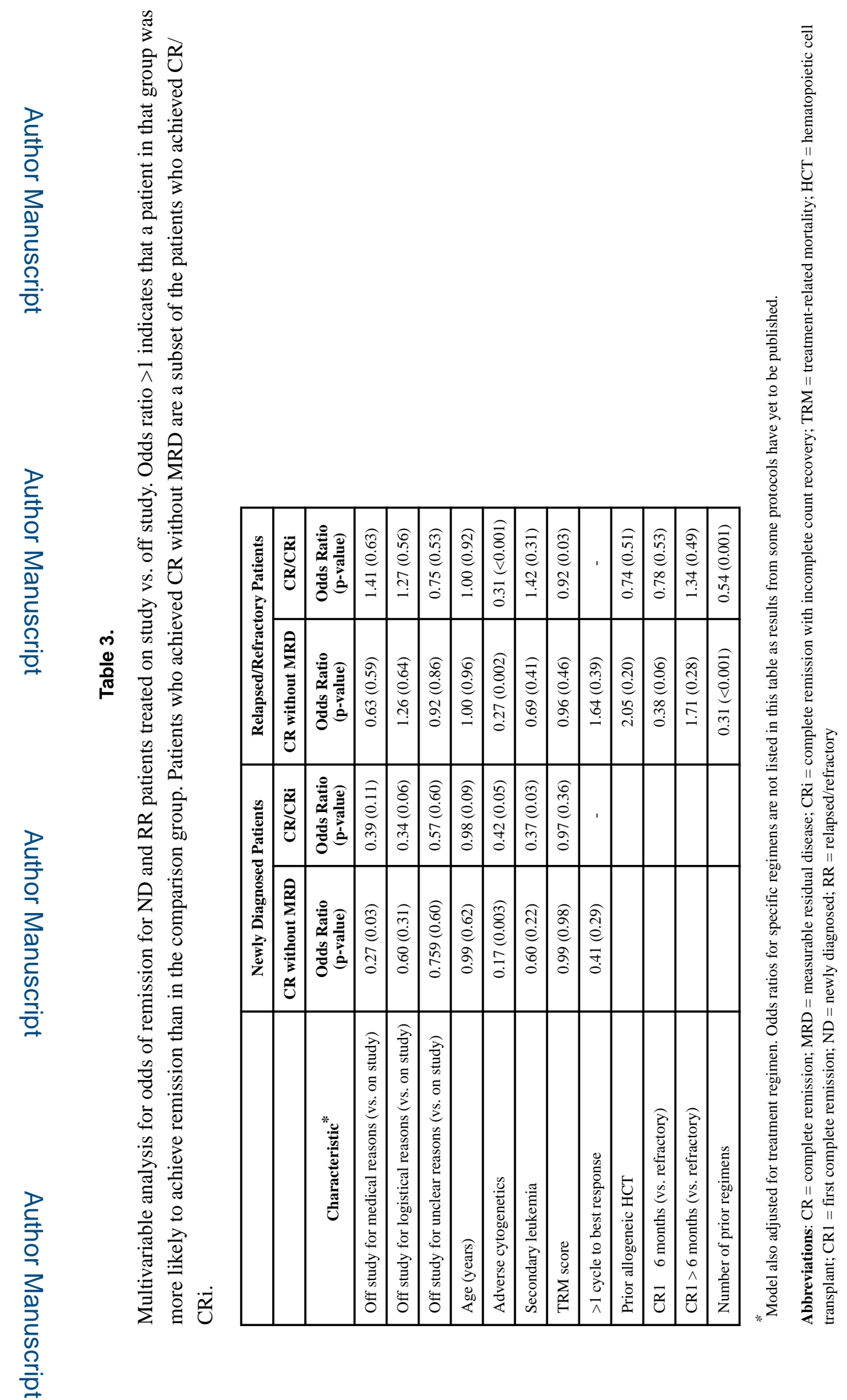

Leuk Lymphoma. Author manuscript; available in PMC 2020 April 01. 


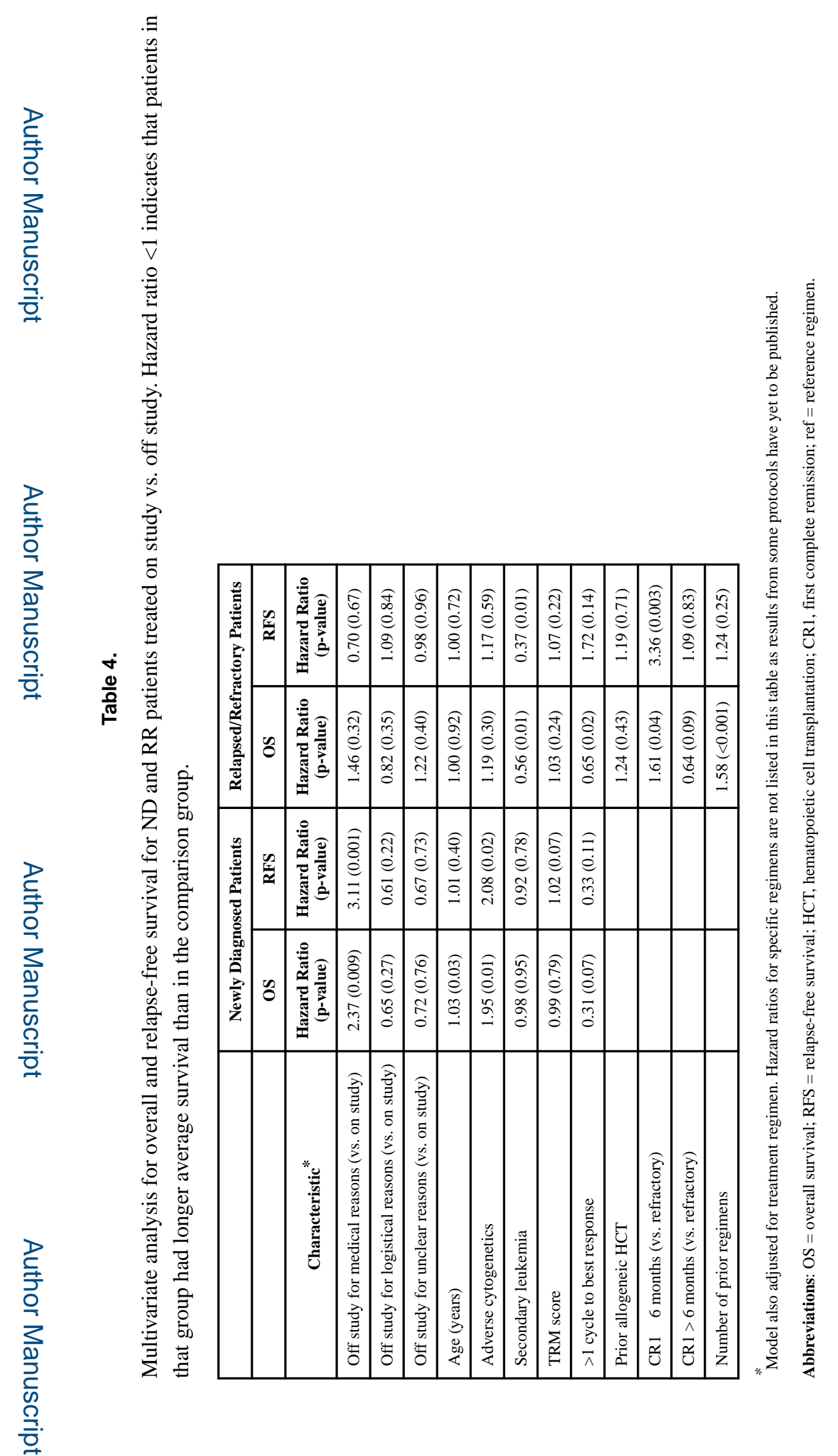

Leuk Lymphoma. Author manuscript; available in PMC 2020 April 01. 